\title{
Characterization of cultured cholangiocytes isolated from livers of patients with primary sclerosing cholangitis
}

\author{
James H Tabibian ${ }^{1,2,4}$, Christy E Trussoni ${ }^{1,2,4}$, Steven P O'Hara ${ }^{1,2}$, Patrick L Splinter ${ }^{1,2}$, Julie K Heimbach ${ }^{3}$ and \\ Nicholas F LaRusso ${ }^{1,2}$
}

Primary sclerosing cholangitis (PSC) is a chronic, idiopathic cholangiopathy. The role of cholangiocytes (biliary epithelial cells) in PSC pathogenesis is unknown and remains an active area of research. Here, through cellular, molecular and nextgeneration sequencing (NGS) methods, we characterize and identify phenotypic and signaling features of isolated PSC patient-derived cholangiocytes. We isolated cholangiocytes from stage 4 PSC patient liver explants by dissection, differential filtration and immune-magnetic bead separation. We maintained cholangiocytes in culture and assessed for: (i) cholangiocyte, cell adhesion and inflammatory markers; (ii) proliferation rate; (iii) transepithelial electrical resistance (TEER); (iv) cellular senescence; and (v) transcriptomic profiles by NGS. We used two well-established normal human cholangiocyte cell lines (H69 and NHC) as controls. Isolated PSC cells expressed cholangiocyte (eg, cytokeratin 7 and 19) and epithelial cell adhesion markers (EPCAM, ICAM) and were negative for hepatocyte and myofibroblast markers (albumin, $\alpha$-actin). Proliferation rate was lower for PSC compared with normal cholangiocytes (4 vs 2 days, respectively, $P<0.01$ ). Maximum TEER was also lower in PSC compared with normal cholangiocytes (100 vs $\left.145 \Omega \mathrm{cm}^{2}, P<0.05\right)$. Interleukin-6 (IL-6) and IL-8 (protein and mRNA) were both increased compared with NHCs and H69s (all $P<0.01$ ). The proportion of cholangiocytes staining positive for senescence-associated $\beta$-galactosidase was higher in PSC cholangiocytes compared with NHCs (48\% vs 5\%, $P<0.01)$. Finally, NGS confirmed cholangiocyte marker expression in isolated PSC cholangiocytes and extended our findings regarding pro-inflammatory and senescence-associated signaling. In conclusion, we have demonstrated that high-purity cholangiocytes can be isolated from human PSC liver and grown in primary culture. Isolated PSC cholangiocytes exhibit a phenotype that may reflect their in vivo contribution to disease and serve as a vital tool for in vitro investigation of biliary pathobiology and identification of new therapeutic targets in PSC. Laboratory Investigation (2014) 94, 1126-1133; doi:10.1038/labinvest.2014.94; published online 21 July 2014

Primary sclerosing cholangitis (PSC) is a chronic, complex, idiopathic disease of the bile ducts (ie, cholangiopathy) characterized by biliary inflammation, periductal fibrosis and cholestasis. ${ }^{1-3}$ Patients with PSC are at increased risk of end-stage cirrhosis as well as hepatobiliary and colonic neoplasia. ${ }^{4,5}$ To date, no effective therapeutic strategies exist for PSC aside from liver transplantation, which itself is associated with numerous challenges, including recurrent PSC and/or cholangiocarcinoma post-transplantation. ${ }^{2,6-8}$ Current animal models of PSC exhibit some of the key features of the human disease (eg, 'onionskin' periductal fibrosis and multifocal strictures) and are useful in studying disease progression; ${ }^{9-11}$ however, no single model reflects the multiple and complex features of PSC or the putative mechanisms involved in the etiopathogenesis of human PSC. ${ }^{12-14}$ Therefore, establishment of primary human cholangiocyte cell cultures from PSC livers would represent an essential tool to facilitate mechanistic, in vitro investigations of the PSC cholangiocyte phenotype and advance current understanding of PSC. ${ }^{15}$

Although representing only $3 \%$ of the total liver cell population, previous reports have described isolation and

\footnotetext{
Division of Gastroenterology and Hepatology, Mayo Clinic College of Medicine, Rochester, MN, USA; ${ }^{2}$ Center for Cell Signaling in Gastroenterology, Mayo Clinic College of Medicine, Rochester, MN, USA and ${ }^{3}$ Division of Transplantation Surgery, Mayo Clinic, Rochester, MN, USA

Correspondence: Dr NF LaRusso, MD, Division of Gastroenterology and Hepatology; Center for Cell Signaling in Gastroenterology, Mayo Clinic College of Medicine, 200 First Street SW, 1701 Guggneheim Building, Rochester, MN 55905, USA.

E-mail: larusso.nicholas@mayo.edu

${ }^{4}$ Co-first authors

Received 12 March 2014; revised 3 June 2014; accepted 10 June 2014
} 
culture of (normal) biliary epithelial cells (ie, cholangiocytes). Isolating and culturing cholangiocytes from PSC liver, however, poses considerable challenges given the cholangiocyte injury, periductal fibrosis and ductopenia inherent to the disease, and to date there are no validated methods to do so. ${ }^{1,2,16-18}$ Our objectives in this study were to: (i) establish methods for high-yield isolation of cholangiocytes from explanted liver from patients with PSC using serial proteinase and hyaluronidase digestion, filtration and immunomagnetic bead purification; (ii) culture and extensively characterize the isolated cells to confirm high expression of cholangiocytespecific markers; and (iii) assess features of PSC and cholangiocyte injury as previously defined by our laboratory and others, including cellular senescence and the senescenceassociated secretory phenotype (SASP). ${ }^{16,19-21}$ Our methodology allows high-purity (99\%) isolation of PSC cholangiocytes, which appear to exhibit characteristics reflective of PSC pathobiology, including the recently appreciated phenomenon of cholangiocyte senescent in ex vivo PSC liver tissue, and that the establishment of these PSC primary cholangiocyte isolates will be a valuable tool for studying the pathogenesis of PSC.

\section{MATERIALS AND METHODS Cell Isolation}

Cells were isolated from liver explant tissue from a 46-yearold male patient with stage 4 PSC without cholangiocarcinoma through a series of digestion, filtration and bead isolations steps. First, the explant tissue was cut into small, easily digestible pieces using sterile razor blades and then incubated in Dulbecco's modified Eagle's medium (DMEM) solution containing fetal bovine serum, penicillin/streptomycin, bovine serum albumin, collagenase and DNase for $45 \mathrm{~min}$ in a shaking water bath at $37^{\circ} \mathrm{C}$. The digested tissue was filtered through a $100 \mu \mathrm{M}$ cell strainer with a subsequent filtration through a $40 \mu \mathrm{M}$ cell strainer. Cells contained in the $40 \mu \mathrm{M}$ cell strainer were washed with DMEM, and subjected to further digestion with a DMEM solution containing hyaluronidase for $30 \mathrm{~min}$ at $37^{\circ} \mathrm{C}$. The resulting hepatic digestant was filtered as described above, and the isolated cells were plated on collagen-coated flasks (BD Biosciences, San Jose, CA, USA) and allowed to grow to confluence. After reaching confluence, cholangiocyte cells were bead isolated using the Epithelial Enrich magnetic bead isolation kit following the manufacturer's instructions (Life Technologies, Grand Island, NY, USA). Of note, cells were also isolated and purified using the same techniques from liver explant tissue from a 58-year-old female and a 57-year-old male patient, both with stage 4 PSC without cholangiocarcinoma, for validation of findings.

\section{Cell Culture}

H69 cells, an SV40-transformed (ie, immortalized) normal human cholangiocyte (NHC) cell line, and low passage number $\mathrm{NHCs}^{22}$ were grown in $\mathrm{H} 69$ media as previously described. PSC cells were grown in media containing DMEM/ F12 (Sigma-Aldrich, St Louis, MO, USA) supplemented with fetal bovine serum (CellGro, Manassas, VA, USA), penicillin/ streptomycin, vitamin solution, MEM solution, CD lipid concentrate, L-glutamine, soybean trypsin inhibitor, insulin/ transferrin/selenium-A, bovine pituitary extract, epidermal growth factor, 3,3'5-triiodo-L-thyronine, dexamethasone and forskolin.

\section{Polymerase Chain Reaction (PCR)}

RNA was isolated from primary PSC cells using TRIzol reagent (Life Technologies), and cDNA was synthesized from the RNA using First Strand cDNA Synthesis kit (Life Technologies). PCR was performed on PSC cDNA for cholangiocyte markers (CK7, CK19, GGT, AQP1 and CFTR), cell adhesion molecules (EPCAM, ICAM and NCAM, inflammatory markers (interleukin (IL)-6 and 8), and noncholangiocyte markers (albumin, $\alpha$-smooth muscle actin). Primer sequences used are provided in Supplementary Table 1.

\section{Immunofluorescence}

H69 and NHC cells were plated on plastic chamber slides and PSC cells were plated on collagen-coated chamber slides (BD Biosciences) and allowed to grow to confluence. Cells were fixed in a $2 \%$ paraformaldehyde solution containing $0.1 \mathrm{M}$ PIPES, $1 \mathrm{mM}$ EGTA and $3 \mathrm{mM} \mathrm{MgSO}$ for $10 \mathrm{~min}$ at room temperature. Cells were washed twice in $1 \mathrm{X}$ phosphate-buffered saline (PBS) and permeabilized with $0.1 \%$ Triton-x 100 in $1 \mathrm{X}$ PBS for $10 \mathrm{~min}$ at room temperature. Cells were then blocked in a $5 \%$ goat serum and $1 \%$ BSA solution for $1 \mathrm{~h}$ at room temperature, washed and incubated overnight at $4{ }^{\circ} \mathrm{C}$ with primary antibodies against CK19 (Sigma) and zonula occludens 1 (ZO1) (Invitrogen, Grand Island, NY, USA). Cells were then washed in PBS plus $0.1 \%$ Tween-20 and incubated with corresponding fluorophore-conjugated goat secondary antibodies (Invitrogen) for $1 \mathrm{~h}$ at room temperature. Cells were washed as before with a final wash in 1X PBS alone. Coverslips were mounted on the cells using Prolong Gold Antifade Reagent with DAPI (Life Technologies). Immunofluorescence was visualized using laser-scanning confocal microscopy (Zeiss, Oberkochen, DE, USA).

\section{Enzyme-Linked Immunosorbant Assay (ELISA)}

Basal IL-6 and IL-8 protein levels were measured from PSC, H69 and NHC cell media supernatants using IL-6 and IL-8 Direct ELISA kits (Invitrogen) following the manufacturer's protocol. Briefly, all cells were grown until they reached confluence, and then media were removed, cells were washed in 1X PBS and incubated in serum-free and epithelial growth factor-free media for $24 \mathrm{~h}$ before samples being collected. Samples were centrifuged for $10 \mathrm{~min}$ at $1000 \mathrm{~g}$ at room temperature to remove cellular debris and then added to the ELISA plates. 


\section{Cell Proliferation}

Cell proliferation assays were done on H69, NHC and PSC cells by performing cell counts. Briefly, all cells were plated at a concentration of 100000 cells per well (day 0) in six-well plates. Cells were trypsinized, resuspended in media and counted on days 1-5 post-plating using a Cellometer Automated Cell Counter (Nexcelom Bioscience). Cell population doubling time was calculated using the formula: $\ln$ (\# cells harvested) $-\ln$ (\# cells seeded)/ln2.

\section{Transepithelial Electrical Resistance (TEER) Measurements}

H69, NHC and PSC cells were seeded on six-well, $3.0 \mu \mathrm{m}$ cell culture inserts (BD Bioscience) and TEER measurements were taken using a Millicell-ERS ohm-voltameter (Millipore) every 24-h afterward until TEER readings started to drop indicating cell-death. TEER values were calculated and expressed as $\Omega / \mathrm{cm}^{2}$.

\section{Cytochemical Staining for SA- $\beta$-Galactosidase}

Plated NHCs and isolated PSC cholangiocytes were fixed and stained using the SA- $\beta$-gal cellular senescence assay kit (Cell Biolabs, San Diego, CA, USA) following the manufacturer's protocol and as previously described by us and others. ${ }^{16,23}$ The percentage of SA- $\beta$-gal-positive cholangiocytes was determined using a 20x objective and bright field illumination of five randomly selected areas.

\section{Next-Generation Sequencing (NGS)}

RNA was prepared in triplicate, purified and qualitatively tested by capillary electrophoresis in the Mayo Molecular Biology Core facility as previously described. ${ }^{24}$ Sequencing data generated from the Illumina HiSeq 2000 instrument (Illumina Inc., San Diego, CA, USA) were analyzed using the in-house RNA-seq pipeline, SnowShoes-EX. FASTQ sequence reads were aligned to both the latest available human genome (19) and our in-house exon junction database using Burroughs-Wheeler alignment as previously described. ${ }^{24}$ In the exon junction database, uni-directional combinations of exon junction database for the sequencing length of 50 bases were generated using exon boundaries defined by the refFlat file from the University of California Santa Clara Table Browser. A maximum of two mismatches were allowed for first 32 bases in each alignment, and reads that had $>2$ mismatches or were mapped to multiple genomic locations (alignment score $<4$ ) were discarded. The aligned sequence tags were counted for all the annotated genes/exons.

The raw reads count from each gene were normalized by the total reads of each biological replicate and then standardized to reads per million (gene counts/total counts of each biological replicate $\times 1000000) .{ }^{24}$ For differential gene expression analysis between groups, we first eliminated genes without any reads across all samples. As scaling by total lane counts (eg, RPKM) can bias estimates of differential expression, we used quantile-based normalization on read counts to determine if genes are differentially expressed ${ }^{25}$ with the negative binomial method requiring an adjusted a

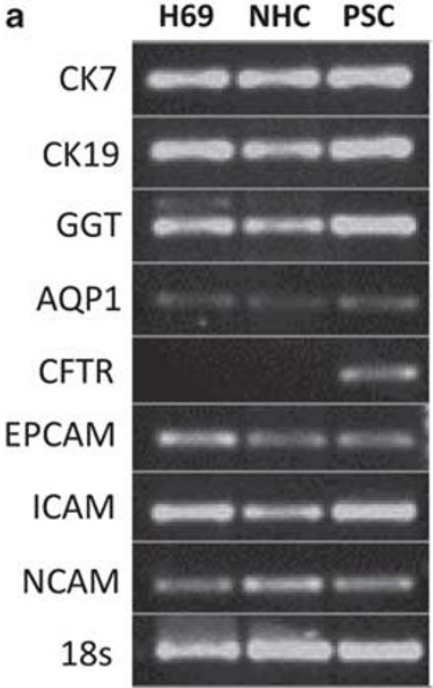

b

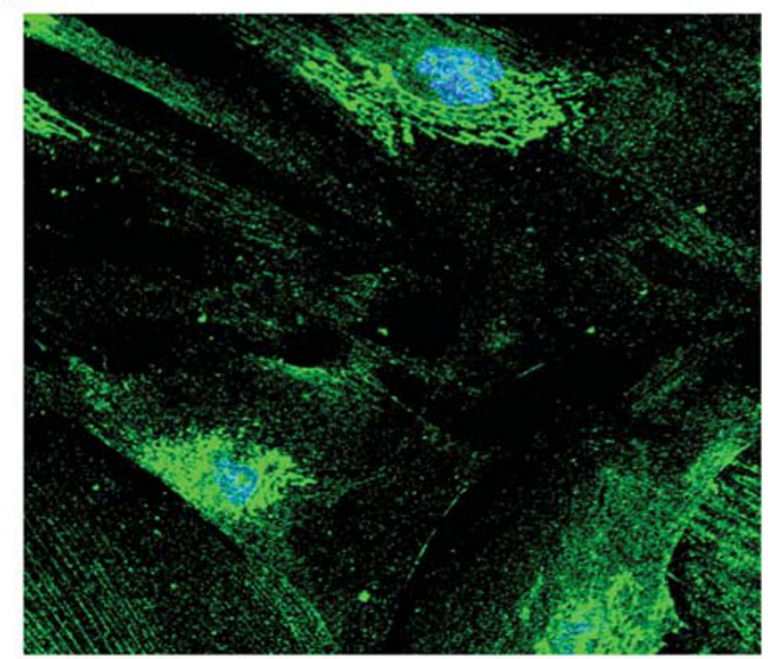

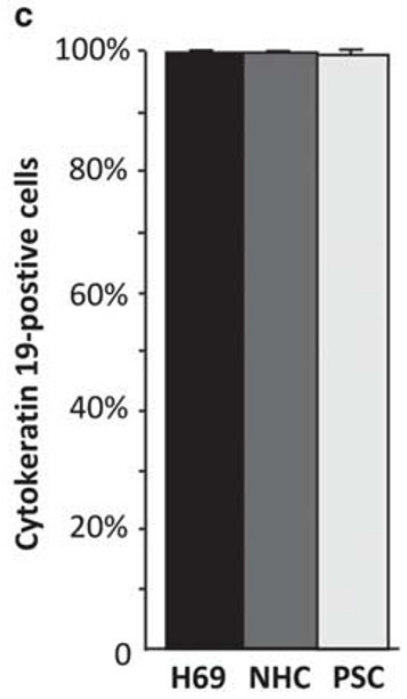

Figure 1 Isolated primary sclerosing cholangitis (PSC) cholangiocytes express biliary epithelial markers at comparable levels to normal cholangiocytes. (a) Isolated PSC cholangiocytes express typical biliary epithelial cell markers CK7, CK19 and GGT in addition to aquaporin and cell adhesion markers that are also expressed in normal cholangiocyte cell lines (semiquantitative PCR). The exception to the similar expression profile between PSC and normal cholangiocytes is CFTR (known to be upregulated in response to liver injury), which is present in PSC cholangiocytes but absent in H69 or normal human cholangiocyte (NHC) cell lines. (b) Isolated PSC cholangiocytes demonstrate CK19 (cholangiocyte marker) positivity as indicated by green immunofluorescence staining. (c) Over $99 \%$ of cholangiocytes isolated from PSC liver explants (as well as established normal cholangiocyte cell lines) express the cholangiocyte marker CK19, indicating a highly purified population of PSC cholangiocytes. 
$P$-value $<0.01$ to control for multiple testing using the Benjamini-Hochberg correction. ${ }^{26}$

\section{RESULTS}

\section{Isolated PSC Cholangiocytes Express Cholangiocyte-} Specific Markers and are Highly Purified

Following size exclusion filtration and epithelium-specific magnetic bead purification of cells isolated from PSC liver (yield $\approx 5 \times 10^{5}$ cells/g of liver tissue), we first assessed cholangiocyte marker expression to confirm the presence of cholangiocytes. We found that isolated PSC cells express the cholangiocyte-specific marker, CK19, as assessed by semiquantitative PCR (Figure 1a) and immunofluorescence microscopy (Figure 1b), in addition to other markers typical of NHCs at levels comparable to established NHC cell lines.
Quantitation of the proportion of cultured cells expressing CK19, as determined by immunofluorescence staining, revealed that $>99 \%$ of the isolated PSC cells were CK19 positive, indicating a highly purified population of PSC-derived intrahepatic cholangiocytes (Figure 1c). We further corroborated these findings by assessing for hepatocyte (albumin) and myofibroblast ( $\alpha$-actin) markers by PCR and immunofluorescence microscopy; both markers were undetectable in isolated PSC cholangiocytes as well as in normal cholangiocyte cell lines (data not shown).

\section{Isolated PSC Cholangiocytes Exhibit Abnormal Cell Morphology and Impaired Epithelial Junctions}

We next assessed morphological characteristics of isolated PSC cholangiocytes compared with NHCs. Compared with
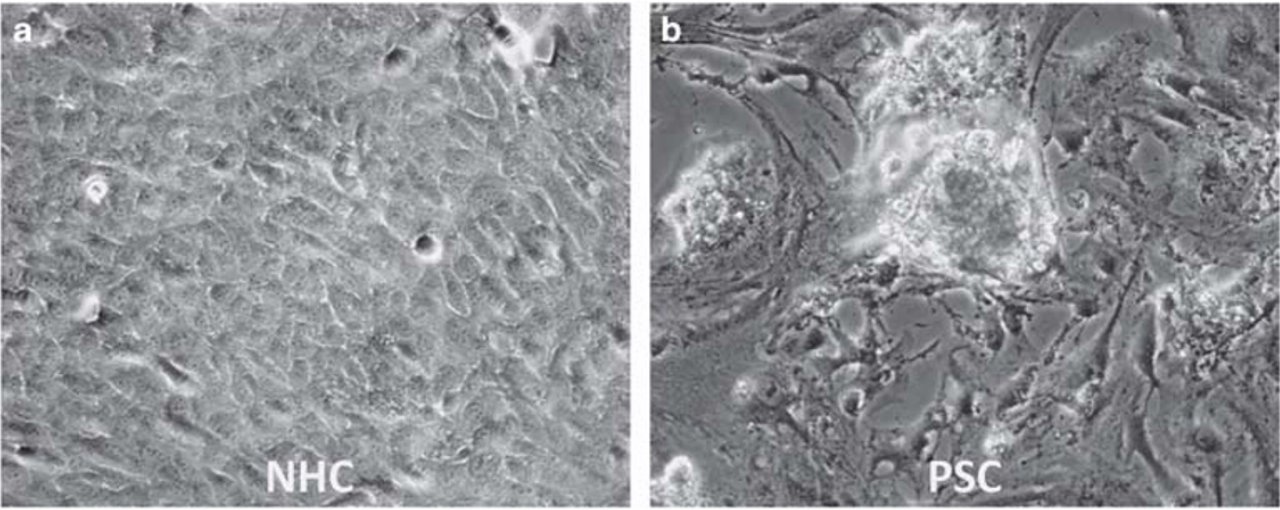
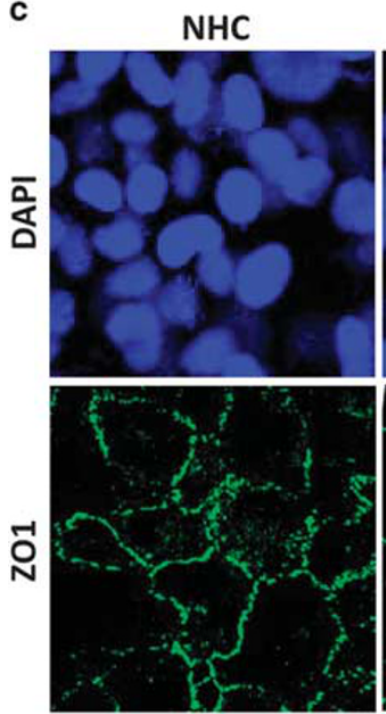

PSC

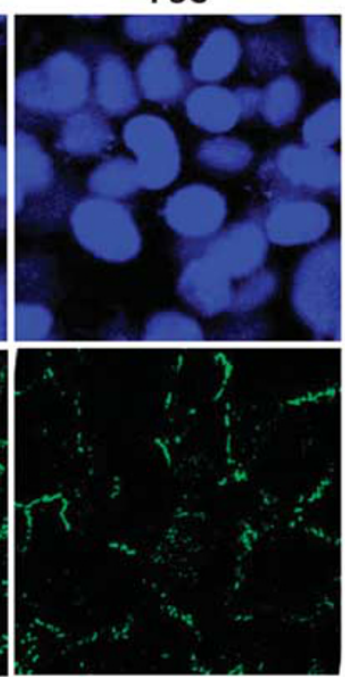

d

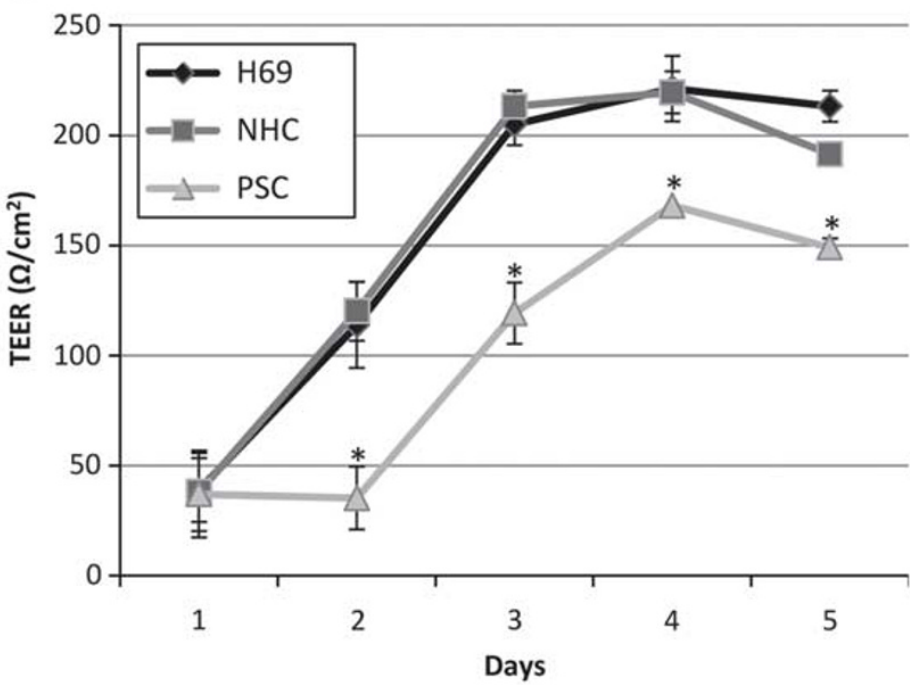

Figure 2 Isolated primary sclerosing cholangitis (PSC) cholangiocytes exhibit abnormal morphological features, diminished tight junction protein expression and impaired barrier integrity. (a) Confluent normal human cholangiocyte (NHC) cell line form a tight monolayer after 7 days in culture, as compared with isolated PSC cholangiocytes (b), which are slow to become confluent after 7 days in culture and appear irregular and larger in size as compared with NHCs (both $\times 20$ magnification). (c) Cultured NHCs form tight junctions with neighboring cells, as indicated by expression of tight junction marker, Z01 (green), whereas cultured PSC cholangiocytes display fewer than normal tight junctions between cells as indicated by decreased ZO1 expression. (d) Transepithelial electrical resistance (TEER) was measured in normal cholangiocytes, H69 and NHC, and isolated PSC cholangiocytes to determine formation and integrity of tight junctions between cells. Isolated PSC cholangiocytes exhibit significantly lower TEER readings compared with both $\mathrm{H} 69$ and $\mathrm{NHC}$ cell lines from days 2 to $5(P<0.01)$, indicating impaired tight junctions in the former. 

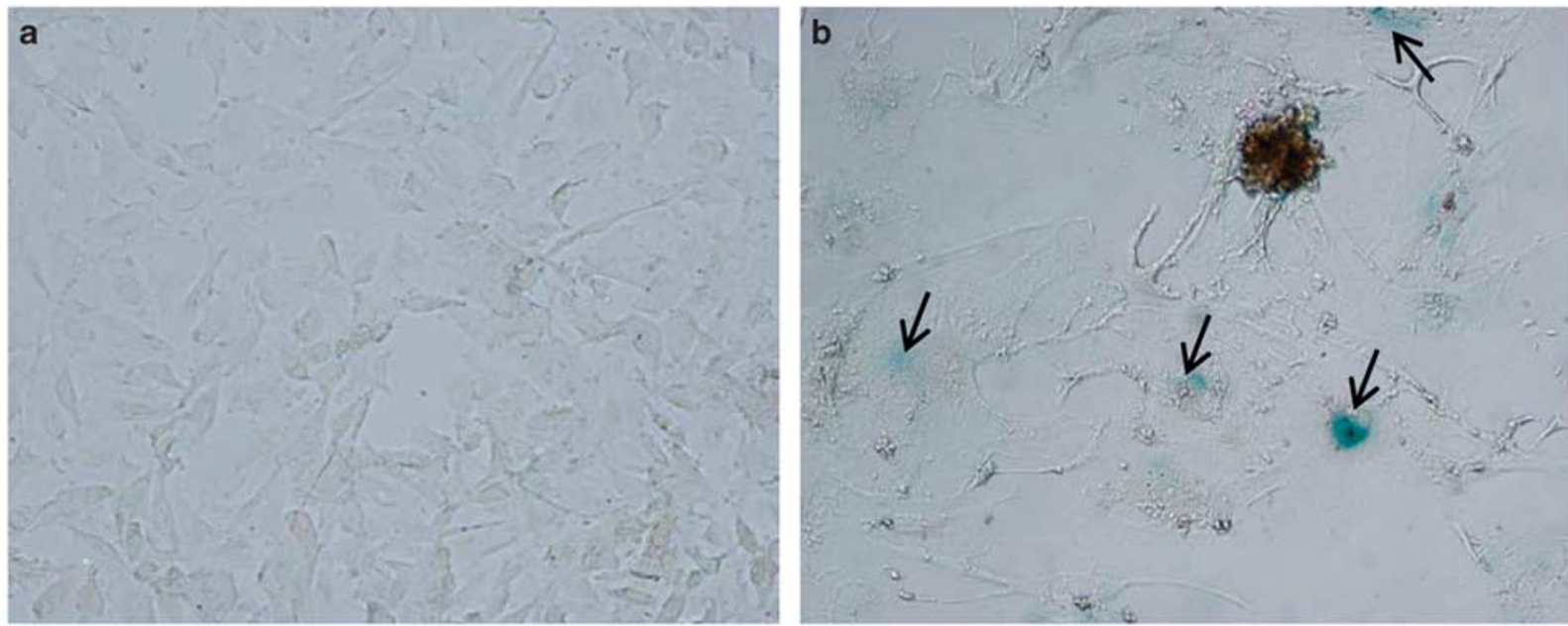

C

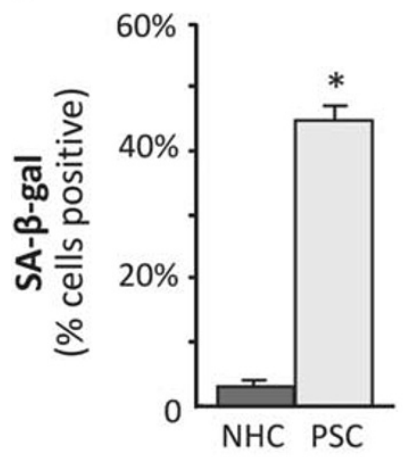

d

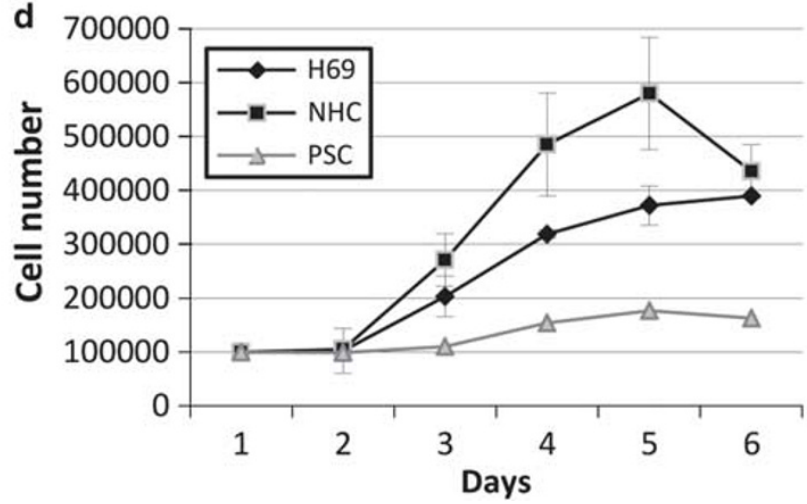

e

\begin{tabular}{|l|c|}
\hline & $\begin{array}{c}\text { Doubling } \\
\text { time } \\
\text { (hours) }\end{array}$ \\
\hline $\mathrm{H} 69$ & 21.7 \\
\hline $\mathrm{NHC}$ & 24.2 \\
\hline $\mathrm{PSC}$ & $75.3^{*}$ \\
\hline *p<0.05 vs. H69 \& NHC
\end{tabular}

Figure 3 Isolated primary sclerosing cholangitis (PSC) cholangiocytes demonstrate features of cellular senescence. Normal human cholangiocytes (NHCs; a) and PSC cholangiocytes (b) were stained (blue) for SA- $\beta$-gal expression, a marker of cellular senescence. (c) Quantitation of blue-stained cells reveals a significantly higher proportion of SA- $\beta$-gal-positive cholangiocytes in PSC compared with normal $(P<0.001)$. (d) Cell counting demonstrates that isolated PSC cholangiocytes have a slower growth rate as compared with NHC and H69 cells. (e) Doubling times of isolated PSC cholangiocytes compared with $\mathrm{NHC}$ and $\mathrm{H} 69$ cells.

NHCs (Figure 2a), we found that isolated PSC cholangiocytes (Figure 2b) exhibit multiple distinguishing features, including enlarged size and robust filamentous (cytoskeletal) structures throughout the cell body. Given these differences, we next assessed whether isolated PSC cholangiocytes achieve the same level of $\mathrm{ZO} 1$ tight junction protein expression. Based on ZO1 immunofluorescence staining, we found that PSC cholangiocytes express less ZO1 protein compared with normal cholangiocytes (Figure 2c). Moreover, based on TEER readings between days 2 and 5 post-plating, we found resistance to be significantly lower $(P<0.01)$ in isolated PSC compared with normal cholangiocytes (Figure 2c).

\section{Isolated PSC Cholangiocytes Exhibit Characteristics of Cellular Senescence}

Given the above findings that isolated PSC cholangiocytes exhibit an enlarged cell size, abnormal cell morphology and impaired ability to form tight junctions or establish a mono- layer, we next evaluated whether these cells express markers of cellular senescence, which we recently described as characteristic of PSC cholangiocytes in vivo. ${ }^{16}$ Low passage isolated PSC cholangiocytes (passage number $\leq 5$ ) and NHCs (passage number $\leq 13$ ) were stained for SA- $\beta$-gal expression, an established marker of cellular senescence. Although NHC cells exhibited minimal blue SA- $\beta$-gal staining (Figure 3a), PSC cholangiocytes exhibited a high proportion of blue SA- $\beta$-gal-stained cells (Figure $3 \mathrm{~b}$ ). Quantitation of SA- $\beta$-gal staining revealed a significantly higher expression of this senescence marker in isolated PSC cholangiocytes compared with NHCs (Figure 3c), suggesting that cultured PSC cholangiocytes maintain the senescent phenotype seen in vivo. We next measured cell proliferation to identify additional potential features of cellular senescence. We found that PSC cholangiocytes exhibited decreased proliferation as compared with normal cholangiocytes (Figures $3 \mathrm{~d}$ and e), with a cell doubling time of $75.3 \mathrm{~h}$ compared with 21.7 and $24.2 \mathrm{~h}$, respectively, for 
H69 and NHC cells, which is congruent with the high proportion of senescent (SA- $\beta$-gal-positive) PSC cholangiocytes.

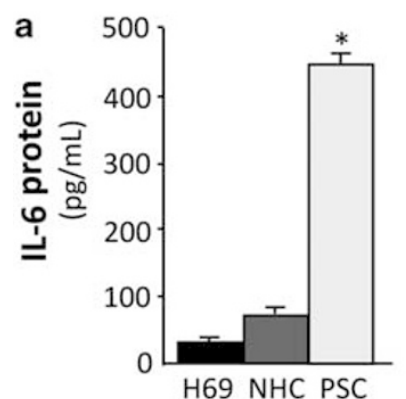

C

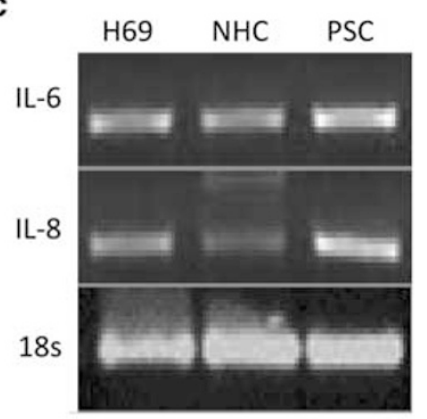

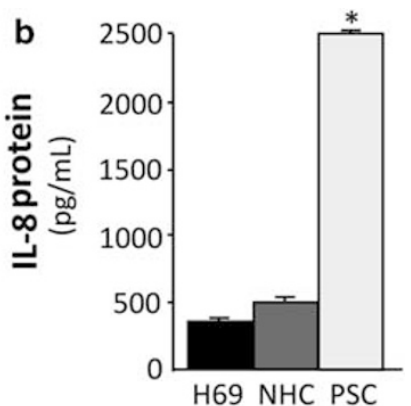

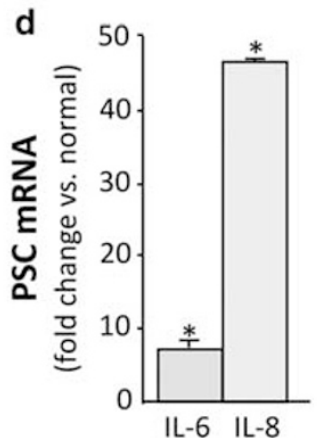

Figure 4 Isolated primary sclerosing cholangitis (PSC) cholangiocytes express increased levels of inflammatory markers. (a) Unstimulated PSC cells secrete significantly higher amounts of the cytokine, IL-6, (up to a ninefold difference) compared with unstimulated $\mathrm{H} 69$ and NHC cells as determined by enzyme-linked immunosorbent assay (ELISA) analysis on cell culture supernate. (b) PSC cells also have a significantly higher basal secretion of the chemokine, interleukin-8 (IL-8), as compared with H69 (a 10 -fold difference) and normal human cholangiocyte (NHC) cells (a fivefold difference). (c) Semiquantitative PCR demonstrates an increase in IL-6 and IL-8 mRNA expression in the PSC cells over $\mathrm{H} 69$ and NHC cells. (d) Quantitative PCR confirms message increase by showing a 20 -fold increase in IL-6 mRNA expression and a 45-fold increase in IL-8 mRNA expression in PSC vs NHCs in a basal state. ${ }^{*} P<0.05$ compared to normal control(s).

Figure 5 Next-generation sequencing (NGS) confirms cholangiocyte marker expression and extends findings regarding the pro-

fibroinflammatory and senescence-associated features of isolated primary sclerosing cholangitis (PSC) cholangiocytes. We examined transcriptomic profiles in PSC cholangiocytes derived from three separate livers as well as $\mathrm{H} 69$ and normal human cholangiocyte (NHC) controls by highthroughput NGS. (a) Cholangiocyte cytoskeletal markers (CK7, $\beta$-actin) are abundantly expressed in both PSC cholangiocytes and normal cholangiocyte cell lines, whereas non-cholangiocyte markers ( $\alpha$-actin, albumin) are minimally expressed, consistent with an enriched cholangiocyte population. (b) Corroborating the immunofluorescence, PCR, and enzyme-linked immunosorbent assay (ELISA) findings, NGSbased mRNA expression of multiple inflammatory cytokines and chemokines is markedly increased in isolated PSC cholangiocytes compared with controls. (c) Markers of senescence (p21) and SASP (PAI-1, IGFBP5 and MMP3) are consistently increased in isolated PSC cholangiocytes. Key: PSC1, PSC2 and PSC3 correspond to a 46-year-old male, 58-year-old female and 57-year-old male patient with PSC, respectively, as described in the Materials and Methods section.

\section{Isolated PSC Cholangiocytes Exhibit Features of the SASP}

To determine whether cultured PSC cholangiocytes also express SASP markers, we measured levels of secreted IL-6 and IL-8, known SASP markers, in cell culture supernatant from isolated PSC cholangiocytes and NHC and H69 cells. Based on ELISA analysis, we found that isolated PSC cholangiocytes secreted significantly higher levels of IL- 6 and IL-8 than both $\mathrm{H} 69$ and NHC cells (Figures $4 \mathrm{a}$ and b). These
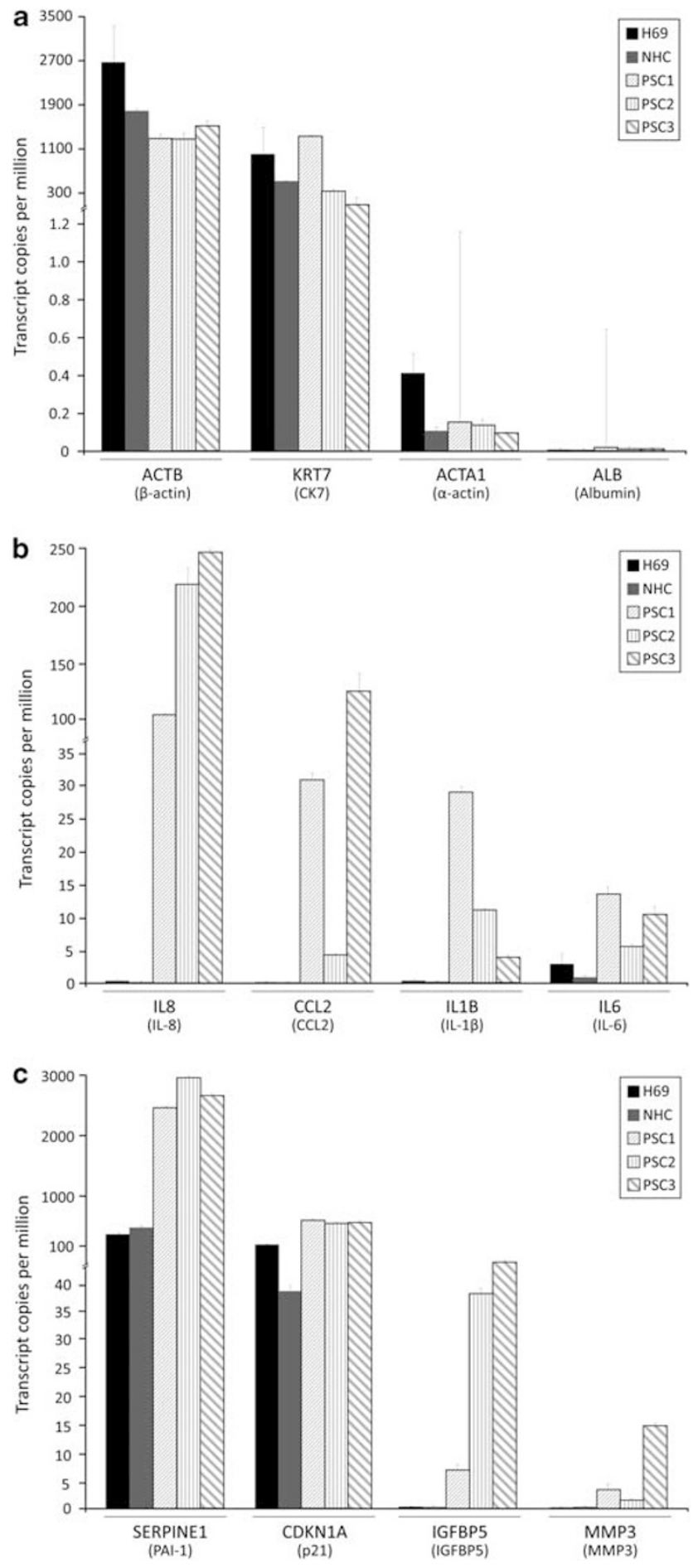
data were corroborated by semiquantitative PCR (Figure 4c) and quantitative PCR (Figure 4d) demonstrating that IL-6 and IL- 8 mRNA levels were increased in isolated PSC compared with normal cholangiocytes, with a 23 -fold change increase in IL-6 and 46-fold increase in IL-8.

\section{NGS Confirms Cholangiocyte Features as well as Pro-Inflammatory Signaling and Features of Cellular Senescence and SASP}

In our last set of experiments, we determined transcriptomic profiles in isolated PSC cholangiocytes, as well as H69 and NHC controls by high-throughput NGS with an emphasis on: (i) cytoskeletal markers, (ii) pro-inflammatory cytokines and chemokines and (iii) senescence markers. In isolated PSC cholangiocytes as well as NHC cell lines, cholangiocyte markers (CK7, $\beta$-actin) were abundantly expressed, whereas hepatocyte and myofibroblast markers (albumin, $\alpha$-actin) were minimally expressed (Figure 5a), thus confirming highpurity cholangiocytes. We next investigated inflammatory markers, and corroborating our immunofluorescence, PCR, and ELISA findings, we observed markedly increased mRNA expression of multiple inflammatory cytokines and chemokines (Figure 5b). Finally, we assessed expression of established senescence (p21) and SASP component (SERPINE1, IGFBP5 and MMP3) gene transcripts; these were consistently increased in isolated PSC cholangiocytes compared with controls (Figure 5c).

\section{DISCUSSION}

In this study, we have described methods to successfully isolate and culture PSC patient-derived cholangiocytes, and, through a combination of cellular, molecular and NGS approaches, we have: (i) verified their purity; (ii) characterized their morphologic and cytologic features; and (iii) identified signaling molecules and pathways relevant to the pathobiology of PSC. Although isolated PSC cholangiocytes express normal cholangiocyte markers (eg, CK7, CK19), they differ significantly from NHC controls in several aspects, including diminished monolayer formation, barrier function and associated tight junction protein expression, as well as impaired growth, upregulation of inflammatory signaling molecules and increased cellular senescence and SASP.

The methods we used to isolate PSC cholangiocytes consisted of both size exclusion filtration and immunomagnetic bead separation. This combined technique is simple, rapid and yields high-purity cholangiocytes with a yield (per gram of liver tissue) similar to other biliary disease conditions; ${ }^{27}$ moreover, immunomagnetic bead separation can be repeated, if needed, to further enrich the cholangiocyte population after several passages. Although other methods of cholangiocyte isolation have been described, for example, perfusate techniques, these are generally cumbersome, costly and/or require whole intact or large segments of liver. Furthermore, these techniques often require considerable purification by differential density centrifugation or elutriation before obtaining a cholangiocyte-enriched cell population yet do not offer significant improvements in yield compared with the methods described herein. ${ }^{28-31}$

Although PSC patient-derived cholangiocytes can be grown under different conditions, we observed that they appear to grow best in our cholangiocyte cell culture media, that is, H69 media, which is supplemented with several growth factors and other pro-growth additives; this may be related to their the underlying diseased state and high prevalence of cholangiocyte senescence, a cellular phenotype recently described by our group as being potentially central to the pathogenesis of PSC. ${ }^{16}$ Even in optimized growth conditions, however, they proliferate slowly and demonstrate an impaired ability to form a monolayer, and hence exhibit decreased tight junction formation and barrier integrity. ${ }^{32,33}$ These collective findings regarding isolated PSC cholangiocytes are consistent with their observed in vivo phenotypic characteristics and pathobiology, as PSC is associated with cholestasis, ductopenia and diminished tight junction integrity, and peribiliary matrix remodeling. ${ }^{2,34-36}$ We do acknowledge, though, that we only studied cholangiocytes from intrahepatic ducts, whereas PSC can also affect the extrahepatic biliary tree; although there is functional and embryological heterogeneity between these cell populations ${ }^{37}$ we believe our findings are very relevant to PSC pathobiology (given that characteristic intrahepatic changes are present in all patients with PSC) but would encourage future studies to also examine and characterize extrahepatic cholangiocytes using methodologies similar to those presented herein. Of note, the cholangiocytes studied herein have maintained expression of cholangiocyte markers for up to five passages but likely have a finite lifespan because of cell culture crisis of primary isolates and ultimately replicative senescence; we are currently attempting to immortalize these cells (eg, SV40 transformation) for further studies yet recognize that certain pathogenic mechanisms (ie, senescence) may be lost as a result of immortalization.

In conclusion, although studies of the pathobiology of PSC have previously been hindered by the lack of available, wellcharacterized primary cholangiocytes, here we demonstrate that these important cells can be successfully isolated, enriched and studied ex vivo. These cells represent a useful tool in dissecting how PSC cholangiocytes sense and respond to stimuli involved in cell proliferation, senescence and ductopenia, as well as biliary fibroinflammation, cholestasis and cell-matrix interactions, that is, autocrine and paracrine signaling processes believed to be fundamentally important to the pathogenesis of the cholangiopathies. Therefore, in sum, having this population of cultured cells for study offers a vital avenue for advancing current understanding of PSC and facilitating identification of novel therapies for this lethal disorder.

Supplementary Information accompanies the paper on the Laboratory Investigation website (http://www.laboratoryinvestigation.org) 


\section{ACKNOWLEDGMENTS}

This work was supported by National Institutes of Health Grants Al089713 (to SPO), DK57993 (to NFL), and DK007198 (T32 training grant support for $\mathrm{JHT}$ ) and by PSC Partners Seeking a Cure.

\section{DISCLOSURE/CONFLICT OF INTEREST}

The authors declare no conflict of interest.

1. Chapman R, Fevery J, Kalloo A, et al. Diagnosis and management of primary sclerosing cholangitis. Hepatology 2010;51:660-678.

2. Tabibian $\mathrm{JH}$, Lindor KD. Primary sclerosing cholangitis: a review and update on therapeutic developments. Exp Rev Gastroenterol Hepatol 2013;7:103-114.

3. Gupta A, Bowlus CL. Primary sclerosing cholangitis: etiopathogenesis and clinical management. Front Biosci 2012;4:1683-1705.

4. Shaib YH, Davila JA, McGlynn K, et al. Rising incidence of intrahepatic cholangiocarcinoma in the United States: a true increase? J Hepatol 2004;40:472-477.

5. Levy C, Lindor KD. Primary sclerosing cholangitis: epidemiology, natural history, and prognosis. Semin Liver Dis 2006;26:22-30.

6. Brandsaeter $\mathrm{B}$, Broome $\mathrm{U}$, Isoniemi $\mathrm{H}$, et al. Liver transplantation for primary sclerosing cholangitis in the Nordic countries: outcome after acceptance to the waiting list. Liver Transpl 2003;9:961-969.

7. Ali JM, Bonomo L, Brais $R$, et al. Outcomes and diagnostic challenges posed by incidental cholangiocarcinoma after liver transplantation. Transplantation 2011;91:1392-1397.

8. Landaverde $\mathrm{C}, \mathrm{Ng} \mathrm{V}$, Sato A, et al. De-novo cholangiocarcinoma in native common bile duct remnant following OLT for primary sclerosing cholangitis. Ann Hepatol 2009;8:379-383.

9. Tabibian JH, Macura SI, O'Hara SP, et al. Micro-computed tomography and nuclear magnetic resonance imaging for noninvasive, live-mouse cholangiography. Lab Invest 2013;93:733-743.

10. Fickert $P$, Zollner $G$, Fuchsbichler $A$, et al. Ursodeoxycholic acid aggravates bile infarcts in bile duct-ligated and Mdr2 knockout mice via disruption of cholangioles. Gastroenterology 2002;123:1238-1251.

11. Yamada $\mathrm{S}$, Ishii $\mathrm{M}$, Liang LS, et al. Small duct cholangitis induced by $\mathrm{N}$-formyl L-methionine L-leucine L-tyrosine in rats. J Gastroenterol 1994:29:631-636.

12. Pollheimer MJ, Trauner M, Fickert P. Will we ever model PSC? - "it's hard to be a PSC model!". Clin Res Hepatol Gastroenterol 2011;35:792-804.

13. Weismuller TJ, Wedemeyer J, Kubicka $S$, et al. The challenges in primary sclerosing cholangitis-aetiopathogenesis, autoimmunity, management and malignancy. J Hepatol 2008;48(Suppl 1):S38-S57.

14. Tabibian JH, Talwalkar JA, Lindor KD. Role of the microbiota and antibiotics in primary sclerosing cholangitis. Biomed Res Int 2013;2013:389537.

15. Alpini G, Phillips JO, Vroman B, et al. Recent advances in the isolation of liver cells. Hepatology 1994;20:494-514.

16. Tabibian JH, O'Hara SP, Splinter PL, et al. Cholangiocyte senescence via $\mathrm{N}$-Ras activation is a characteristic of primary sclerosing cholangitis. Hepatology 2014;59:2263-2275.

17. Wiesner RH, LaRusso NF. Clinicopathologic features of the syndrome of primary sclerosing cholangitis. Gastroenterology 1980;79:200-206.

18. Vitellas KM, Keogan MT, Freed KS, et al. Radiologic manifestations of sclerosing cholangitis with emphasis on MR cholangiopancreatography. Radiographics 2000;20:959-975, quiz 1108-9, 1112.
19. O'Hara SP, Splinter PL, Trussoni CE, et al. Cholangiocyte N-Ras protein mediates lipopolysaccharide-induced interleukin 6 secretion and proliferation. J Biol Chem 2011;286:30352-30360.

20. Sasaki $\mathrm{M}$, Ikeda $\mathrm{H}$, Haga $\mathrm{H}$, et al. Frequent cellular senescence in small bile ducts in primary biliary cirrhosis: a possible role in bile duct loss. J Pathol 2005;205:451-459.

21. Karrar A, Broome U, Sodergren T, et al. Biliary epithelial cell antibodies link adaptive and innate immune responses in primary sclerosing cholangitis. Gastroenterology 2007;132:1504-1514.

22. Banales JM, Saez E, Uriz M, et al. Up-regulation of microRNA 506 leads to decreased $\mathrm{Cl}-/ \mathrm{HCO} 3-$ anion exchanger 2 expression in biliary epithelium of patients with primary biliary cirrhosis. Hepatology 2012;56:687-697.

23. Dimri GP, Lee $X$, Basile $G$, et al. A biomarker that identifies senescent human cells in culture and in aging skin in vivo. Proc Natl Acad Sci USA 1995;92:9363-9367.

24. Craig TA, Zhang $Y$, McNulty MS, et al. Research resource: whole transcriptome RNA sequencing detects multiple 1alpha,25dihydroxyvitamin $\mathrm{D}(3)$-sensitive metabolic pathways in developing zebrafish. Mol Endocrinol 2012;26:1630-1642.

25. Bullard JH, Purdom E, Hansen KD, et al. Evaluation of statistical methods for normalization and differential expression in mRNA-Seq experiments. BMC Bioinform 2010;11:94.

26. Anders S, Huber W. Differential expression analysis for sequence count data. Genome Biol 2010;11:R106.

27. Joplin R, Strain AJ, Neuberger JM. Biliary epithelial cells from the liver of patients with primary biliary cirrhosis: isolation, characterization, and short-term culture. J Pathol 1990;162:255-260.

28. Gall JA, Bhathal PS. The isolation of intrahepatic biliary epithelial cells from normal rat livers. Cell Biol Int Rep 1985;9:315-322.

29. Grant AG, Billing BH. The isolation and characterization of a bile ductule cell population from normal and bile-duct ligated rat livers. $\mathrm{Br}$ J Exp Pathol 1977;58:301-310.

30. Joplin R, Strain AJ, Neuberger JM. Immuno-isolation and culture of biliary epithelial cells from normal human liver. In Vitro Cell Dev Biol 1989;25:1189-1192.

31. Alpini G, Lenzi R, Zhai WR, et al. Isolation of a nonparenchymal liver cell fraction enriched in cells with biliary epithelial phenotypes. Gastroenterology 1989;97:1248-1260.

32. Madara JL. Tight junction dynamics: is paracellular transport regulated? Cell 1988;53:497-498.

33. Muff MA, Masyuk TV, Stroope AJ, et al. Development and characterization of a cholangiocyte cell line from the PCK rat, an animal model of autosomal recessive polycystic kidney disease. Lab Invest 2006;86:940-950.

34. Washington K, Clavien PA, Killenberg P. Peribiliary vascular plexus in primary sclerosing cholangitis and primary biliary cirrhosis. Hum Pathol 1997:28:791-795.

35. Fickert $P$, Fuchsbichler $A$, Wagner $M$, et al. Regurgitation of bile acids from leaky bile ducts causes sclerosing cholangitis in Mdr2 (Abcb4) knockout mice. Gastroenterology 2004;127:261-274.

36. O'Hara SP, Tabibian JH, Splinter $\mathrm{PL}$, et al. The dynamic biliary epithelia: molecules, pathways, and disease. J Hepatol 2013;58: 575-582.

37. Han Y, Glaser S, Meng F, et al. Recent advances in the morphological and functional heterogeneity of the biliary epithelium. Exp Biol Med (Maywood) 2013;238:549-565. 